\title{
Genome-wide association study reveals putative role of gga-miR-15a in controlling feed conversion ratio in layer chickens
}

\author{
Jingwei Yuan', Sirui Chen ${ }^{1}$, Fengying Shi ${ }^{2}$ Guiqin $\mathrm{Wu}^{2}$, Aiqiao Liu², Ning Yang ${ }^{1}$ and Congjiao Sun ${ }^{1 *}$
}

\begin{abstract}
Background: Efficient use of feed resources for farm animals is a critical concern in animal husbandry. Numerous genetic and nutritional studies have been conducted to investigate feed efficiency during the regular laying cycle of chickens. However, by prolonging the laying period of layers, the performance of feed utilization in the latelaying period becomes increasingly important. In the present study, we measured daily feed intake $(\mathrm{FI})$, residual feed intake (RFI) and feed conversion ratio (FCR) of 808 hens during 81-82 weeks of age to evaluate genetic properties and then used a genome-wide association study (GWAS) to reveal the genetic determinants.

Results: The heritability estimates for the investigated traits were medium and between 0.15 and 0.28 in both pedigree- and genomic-based estimates, whereas the genetic correlations among these traits were high and ranged from 0.49 to 0.90 . Three genome-wide significant SNPs located on chromosome 1 (GGA1) were detected for FCR. Linkage disequilibrium (LD) and conditional GWA analysis indicated that these 3 SNPs were highly correlated with one another, located at 13.55-45.16 Kb upstream of gga-miR-15a. Results of quantitative real-time polymerase chain reaction (qRT-PCR) analysis in liver tissue showed that the expression of gga-miR-15a was significantly higher in the high FCR birds than that in the medium or low FCR birds. Bioinformatics analysis further revealed that ggamir-15a could act on many target genes, such as forkhead box O1 (FOXO1) that is involved in the insulin-signaling pathway, which influences nutrient metabolism in many organisms. Additionally, some suggestively significant variants, located on GGA3 and GGA9, were identified to associate with FI and RFI.

Conclusions: This GWA analysis was conducted on feed intake and efficiency traits for chickens and was innovative for application in the late laying period. Our findings can be used as a reference in the genomic breeding programs for increasing the efficiency performance of old hens and to improve our understanding of the molecular determinants for feed efficiency.
\end{abstract}

Keywords: Feed efficiency, Genome-wide association study, Gga-miR-15a, Late laying period, Chickens

\section{Background}

People are always paying attention to animal feed efficiency because of the large effect on farm profitability. For the poultry industry, feed efficiency represents its competitive position against other animal protein sources, and to food economists, efficiency places less demand on global feed resources [1]. The advances in optimizing diet formulations have significantly improved

\footnotetext{
* Correspondence: cjsun@cau.edu.cn

${ }^{1}$ National Engineering Laboratory for Animal Breeding and MOA Key

Laboratory of Animal Genetics and Breeding, College of Animal Science and

Technology, China Agricultural University, Beijing, China

Full list of author information is available at the end of the article
}

the feed efficiency for layers in the past decades. However, with increasing feed costs, further improvement by genetics and breeding strategies is a particularly important aspect. By integrating statistical genetics, molecular biology and sequencing technology in numerous studies, the genetic determinants for many economic traits of farm animals have been revealed, such as the blue eggshell in chicken [2], glycogen content of skeletal muscle in pig [3], and pleiotropic polymorphisms for stature, fatness and reproduction traits in beef [4]. Poultry geneticists have focused on elucidating the genetic mechanisms that determine feed efficiency, such as identifying quantitative trait loci (QTLs) and genomic variants in 
chicken [5], waterfowl [6], turkey [7], and quail [8], among others. However, most of the screened loci that putatively influence feed efficiency are breed-, age-, or breeding area-specific (http://www.animalgenome.org/ cgi-bin/QTLdb/GG/index). The previous findings indicate that feed efficiency as a variably quantitative trait requires a more accurate and comprehensive strategy to reveal the genetic factors for birds under several conditions.

In the egg-type chicken industry, a trend has developed to prolong the laying cycle, which is related to animal welfare, the ecological footprint of animal production and the use of natural resources [9]. However, the decline of performance for old hens represents a substantial challenge for this development pattern. Accordingly, to prolong persistent bird performance, feed efficiency must be addressed. To achieve the above goals, genetic determinants for feed efficiency of old hens require investigation. In the current study, feed efficiency traits were measured for layers 81-82 weeks of age, and then the GWAS method with a molecular validation strategy was used to detect the genetic variants and candidate genes that were related to feed efficiency.

\section{Results}

\section{Phenotypic descriptions and genetic properties}

The descriptive statistics of daily feed intake (FI), residual feed intake (RFI), feed conversion ratio (FCR), body weight (MBW) and daily egg mass (EM) are presented in Table 1 for 808 qualified hens. Chickens consumed an average of $122 \mathrm{~g}$ of feed and produced $\sim 50 \mathrm{~g}$ of egg mass per day in the laying period of $81-82$ weeks of age. The minimum and maximum values of RFI were $-41.65 \mathrm{~g} / \mathrm{d}$ and $43.69 \mathrm{~g} / \mathrm{d}$, respectively. The coefficient of variation (CV) of FCR (20.18\%) was higher than that of FI (11\%). The raw data of RFI were normally distributed, and the data of FI and FCR fitted a normal distribution after Johnson transformation.

Estimates of heritability and genetic correlations among these traits are listed in Table 2. Pedigree-based heritability estimates for FI $(0.18 \pm 0.07)$ and RFI $(0.20 \pm 0.07)$ were lower than that for FCR $(0.28 \pm 0.09)$. Compared with pedigree-based estimates, the SNP-based heritability estimates were lower for FI $(0.15 \pm 0.05)$, RFI $(0.17 \pm 0.05)$

Table 1 Descriptive statistics of feed efficiency and related traits ${ }^{a}$

\begin{tabular}{llllll}
\hline Traits $^{\text {b }}$ & Mean & SD & CV(\%) & Min & Max \\
\hline FI (g/d) & 121.87 & 13.41 & 11.00 & 70.70 & 165.06 \\
RFI (g/d) & 0 & 12.47 & - & -41.65 & 43.69 \\
FCR (g:g) & 2.51 & 0.51 & 20.18 & 1.58 & 4.73 \\
EM (g/d) & 48.55 & 8.74 & 17.48 & 15.57 & 71.64 \\
MBW (g) & 2236.9 & 182.9 & 8.16 & 1638.0 & 2936.0 \\
\hline
\end{tabular}

${ }^{a} n=808$
${ }^{b} F_{1}, R F I, F C R, E M$ and MBW represent daily feed intake, residual feed intake and feed conversion ratio, daily egg mass and mean body weight, respectively
Table 2 Genetic parameters for daily feed intake, residual feed intake and feed conversion ratio ${ }^{a}$

\begin{tabular}{llll}
\hline Traits $^{\mathrm{b}}$ & $\mathrm{Fl}$ & $\mathrm{RFI}$ & $\mathrm{FCR}$ \\
\hline $\mathrm{FI}$ & $\mathbf{0 . 1 5 ( \mathbf { 0 . 0 5 } )}$ & $0.86(0.06)$ & $0.39(0.20)$ \\
& $\mathbf{0 . 1 8 ( 0 . 0 7 )}$ & & \\
$\mathrm{RFI}$ & $0.90(0.05)$ & $\mathbf{0 . 1 7 ( 0 . 0 5 )}$ & $0.71(0.13)$ \\
& & $\mathbf{0 . 2 0 ( 0 . 0 7 )}$ & \\
FCR & $0.49(0.22)$ & $0.71(0.15)$ & $\mathbf{0 . 2 1}(\mathbf{0 . 0 5 )}$ \\
& & & $\mathbf{0 . 2 8}(\mathbf{0 . 0 9})$
\end{tabular}

${ }^{a}$ Heritability is given on diagonal (italic bold is pedigree-based heritability and bold is SNP-based), pedigree-based genetic correlations below diagonal and SNP-based genetic correlations above diagonal. Standard errors of estimates are in parentheses

${ }^{\mathrm{b}} \mathrm{Fl}$ : daily feed intake, RFI: residual feed intake, FCR: feed conversion ratio

and FCR $(0.21 \pm 0.05)$. Regarding the genetic correlations, SNP-based estimates were a little different from pedigreebased estimates. The highest correlation was found between FI and RFI $(0.90 \pm 0.05$ and $0.86 \pm 0.06$ for pedigree- and SNP-based estimates, respectively). By contrast, the lowest correlations for pedigree- and SNP-based estimates were found between FI and FCR at 0.49 and 0.39 with substantial standard errors of 0.22 and 0.20 , respectively.

\section{Genome-wide association study}

The Manhattan and quantile-quantile (Q-Q) plots for FCR, FI and RFI are shown in Fig. 1. Genome-wide association analysis revealed 3 genome-wide significant SNPs (Table 3) and 11 suggestively significant SNPs (Additional file 1: Table S1) associated with FCR (Fig. 2a). These SNPs were in high linkage disequilibrium (Fig. 2b) and located in a region that ranged from 168.62 to $168.80 \mathrm{Mb}$ on GGA1. Together, these 14 SNPs explained $2.30 \%$ of the phenotypic variance of FCR. GgamiR-15a (MIR15A) was the only gene near these 3 genome-wide significant loci. Several genes also harbored or were near the 11 suggestively significant SNPs, including gga-miR-16a-1 (MIR16-1), deleted in lymphocytic leukemia 2 (DELU2), SPRY domain containing 7 (SPRYD7), potassium channel regulator (KCNRG) and tripartite motif containing (TRIM13). To further test the possible secondary association signals at the locus, conditional GWA analysis was conducted with the top associated SNP rs13553102 as a covariate. All SNPs were hidden below the suggestively significant line (Additional file 2: Figure S1) after conditional GWA analysis, which suggested that SNP rs13553102 was the most reliable signal in this region. The SNP was located at $13.55 \mathrm{~Kb}$ upstream of MIR15A with a MAF of 0.41. The substitution of variant A to $G$ for $r s 13553102$ led to a significant decrease in FCR value (Fig. 2c). The genomic inflation factor (GIF) was 0.99 for FCR and indicated that 

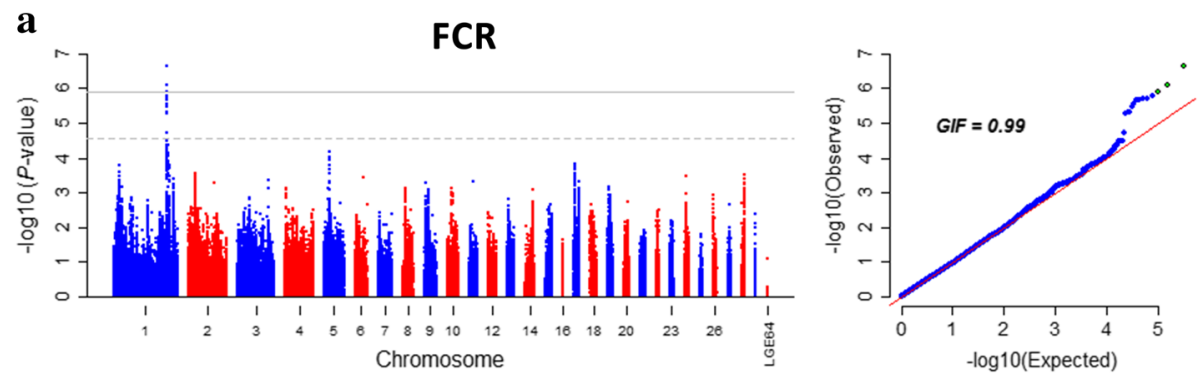

b

FI
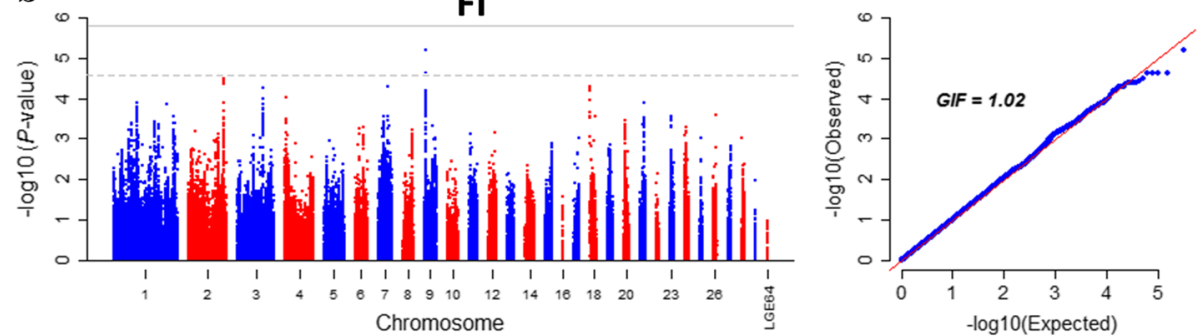

C
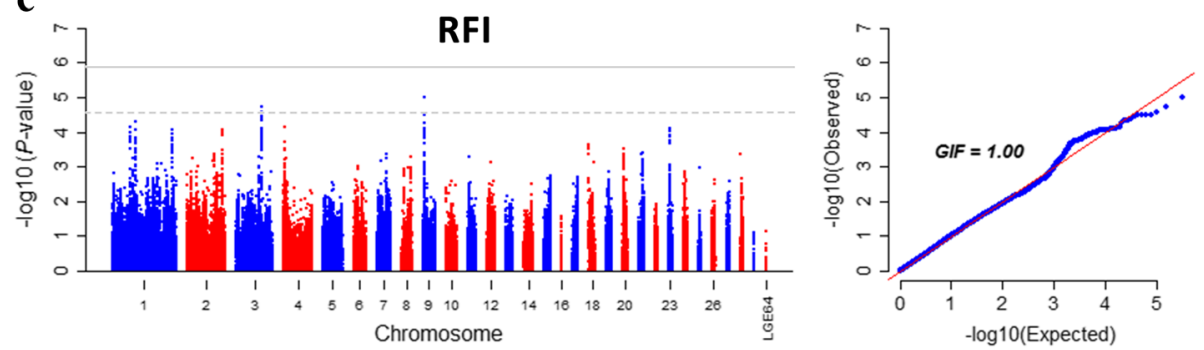

Fig. 1 Manhattan and Q-Q plot of genome wide association study for feed intake and efficiency traits. Each dot represents a SNP in the dataset. The horizontal gray line and gray dashed line indicate the genome-wise significance threshold $(P$ value $=1.29 \mathrm{e}-6)$ and genome-wise suggestive significance threshold ( $P$ value $=2.58 \mathrm{e}-5$ ), respectively. FI, RFI and FCR denote daily feed intake, residual feed intake and feed conversion ratio, respectively. GIF represents genomic inflation factor. a) Plot for feed conversion ratio, b) Plot for daily feed intake, c) Plot for residual feed intake

Table 3 The information for SNPs associated with feed intake and efficiency traits

\begin{tabular}{|c|c|c|c|c|c|c|c|c|}
\hline$\overline{\text { Traits }^{a}}$ & SNP & $\mathrm{GGA}^{\mathrm{b}}$ & Position & $P$-value ${ }^{c}$ & $\mathrm{MAF}^{\mathrm{d}}$ & $\beta^{e}$ & $\begin{array}{l}\text { Candidate/ } \\
\text { nearest gene }\end{array}$ & Location $(\mathrm{kb})^{f}$ \\
\hline \multirow[t]{3}{*}{ FCR } & rs13553102 & 1 & $168,708,318$ & $2.35 \mathrm{e}-7^{*}$ & $0.41(\mathrm{~A} / \mathrm{G})$ & -0.29 & MIR15A & U 13.57 \\
\hline & rs314376310 & 1 & $168,738,343$ & $7.93 e-7^{*}$ & $0.51(\mathrm{G} / \mathrm{C})$ & -0.29 & MIR15A & U 43.57 \\
\hline & rs13972109 & 1 & $168,739,928$ & $1.27 e-6^{*}$ & $0.50(\mathrm{~T} / \mathrm{C})$ & -0.28 & MIR15A & U 45.16 \\
\hline \multirow[t]{5}{*}{$\mathrm{FI}$} & rs313839239 & 9 & $4,521,384$ & $6.21 \mathrm{e}-6$ & $0.06(\mathrm{~T} / \mathrm{C})$ & 0.56 & FARP2 & Intron 1 \\
\hline & rs313750381 & 9 & $4,358,988$ & $2.47 e-5$ & $0.04(\mathrm{~A} / \mathrm{G})$ & 0.59 & KIF1A & Intron 22 \\
\hline & rs314936159 & 9 & $4,371,299$ & $2.47 e-5$ & $0.04(\mathrm{~A} / \mathrm{G})$ & 0.59 & KIF1A & $\cup 0.59$ \\
\hline & rs313292633 & 9 & $4,397,583$ & $2.47 e-5$ & $0.04(\mathrm{~T} / \mathrm{C})$ & 0.59 & SNED1 & Exon 11 \\
\hline & rs312606176 & 9 & $4,402,911$ & $2.47 e-5$ & $0.04(G / A)$ & 0.59 & SNED1 & Intron 22 \\
\hline \multirow[t]{2}{*}{ RFI } & rs314723494 & 3 & $75,533,793$ & $1.94 \mathrm{e}-5$ & $0.33(\mathrm{~T} / \mathrm{C})$ & 3.17 & CNR1 & U 46.60 \\
\hline & rs313839239 & 9 & $4,521,384$ & $1.01 \mathrm{e}-5$ & $0.06(\mathrm{~T} / \mathrm{C})$ & 6.74 & FARP2 & Intron 1 \\
\hline
\end{tabular}

${ }^{\mathrm{a}} \mathrm{FCR}, \mathrm{FI}$ and $\mathrm{RFI}$ represent daily feed intake, residual feed intake and feed conversion, respectively

${ }^{\mathrm{b}}$ Chicken chromosome

${ }^{c *}$ Indicates that the SNP $P$ value reaches a genome-wise significance

${ }^{\mathrm{d}}$ Allele frequency of the first listed marker

eEffect of allele substitution

${ }^{\mathrm{f}} \mathrm{U}$ indicates that the SNP is upstream of a gene $5^{\prime}$-UTR 

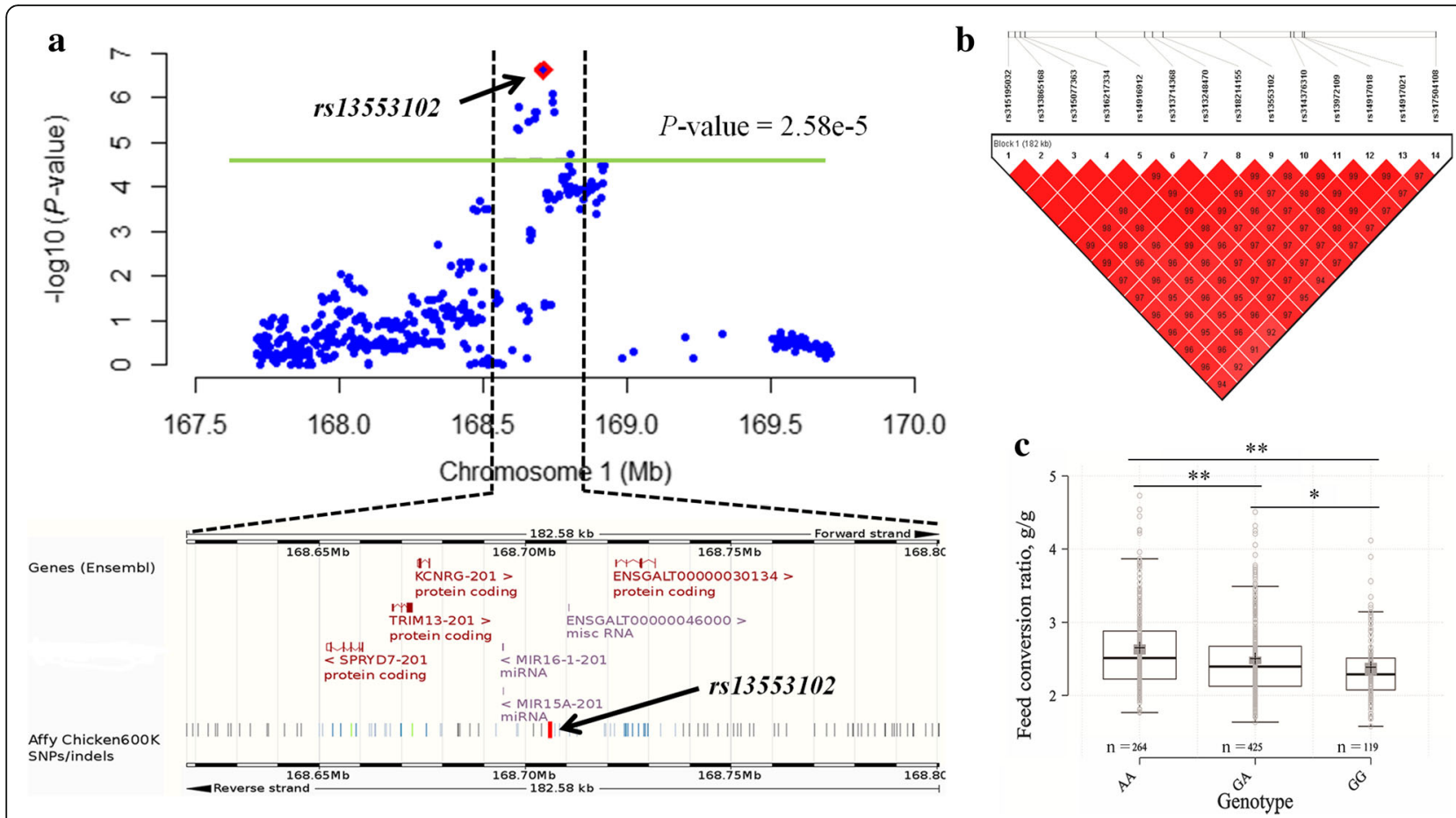

Fig. 2 Association results of candidate region on chromosome 1 (GGA1) for feed conversion ratio (FCR). a Location of the loci associated with FCR on GGA1. The graph plots genomic position ( $x$ axis) against its significance expressed as -log $10 P$ value (y axis). Genomic position of associated SNPs reaching suggestive significance $(P$-value $=2.58 \mathrm{e}-5)$ indicated by a green horizon line span $182.58 \mathrm{~kb}$. The SNP $\mathrm{rs} 13553102 \mathrm{is}$ red highlighted. The annotated candidate genes and SNP displayed below the graph downloaded from Ensembl database. $\mathbf{b}$ Linkage disequilibrium (LD) plot for the 14 SNPs reaching suggestive significance in the candidate region on GGA1. c Genotype effect plot of the SNP rs 13553102. ** $(P<0.01)$ and $*(P<0.05)$ indicate significant differences among groups $(n=264,425$ and 119 for AA, GA and GG, respectively)

the population stratification was well corrected in the analysis.

For FI and RFI, we didn't find any genome-wide significant hit. However, a handful of secondary important SNPs were identified at a suggestively significant level. Five suggestively significant SNPs with a low minor allele frequency (MAF < 0.1 ) were detected for FI on chromosome 9 (GGA9) spanning from 4.36 to $4.52 \mathrm{Mb}$. All SNPs had a positive effect on FI. Linkage disequilibrium (LD) analysis showed that the 5 SNPs were in a high linkage phase (Additional file 3: Figure S2), suggesting that a potential QTL affecting FI might be harbored in this region. The candidate genes in this region included FARP2 (FERM, RhoGEF and pleckstrin domain protein 2), KIF1A (kinesin family member 1A) and SNED1 (sushi, nidogen and EGF-like domains 1). Compared with the FI, only two SNPs, rs314723494 and rs313839239, with genome-wide suggestive significance were detected for RFI. The two SNPs were located on GGA3 and GGA9. The identical SNP rs313839239 identified for FI and RFI might support the high genetic correlation between FI and RFI (Table 2). SNP rs313839239 had a low frequency of minor allele $\mathrm{T}(\mathrm{MAF}=0.06)$ in the current population. However, the substitution of variant $C$ to $T$ caused a significant difference for both FI (Fig. 3a) and RFI (Fig. 3b) values. Another SNP rs314723494 with a positive effect on the RFI was located in the $46.6 \mathrm{~kb}$ upstream of cannabinoid receptor 1 (CNR1). Chickens with the CC genotype of rs314723494 were more efficient with a $-1.88 \mathrm{~g} / \mathrm{d}$ RFI than TC and TT genotypes with 0.27 and $6.53 \mathrm{~g} / \mathrm{d}$ RFI, respectively (Fig. 3c). Moreover, the GIF was 1.02 and 1.00 for FI and RFI, respectively, indicating that the association analyses were scarcely affected by the population stratification.

\section{Expression of gga-miR-15a in liver tissue}

According to the association analysis of FCR, all genome-wide significant SNPs were near gga-miR-15a. We considered gga-miR-15a a promising candidate that might be associated with feed efficiency. Therefore, based on the FCR values (Fig. 4a) only, we selected six birds from high (HFCR), medium (MFCR) and low FCR (LFCR) groups and then extracted the total RNA of their liver tissue. The cDNA was used to run quantitative real-time PCR (qRT-PCR) for gga-miR-15a. We found that the relative expression of gga-miR-15a was significantly higher in the HFCR group than that in MFCR and LFCR groups (Fig. 4b), suggesting that the gga-miR-15a should be a promising candidate gene for feed efficiency. 

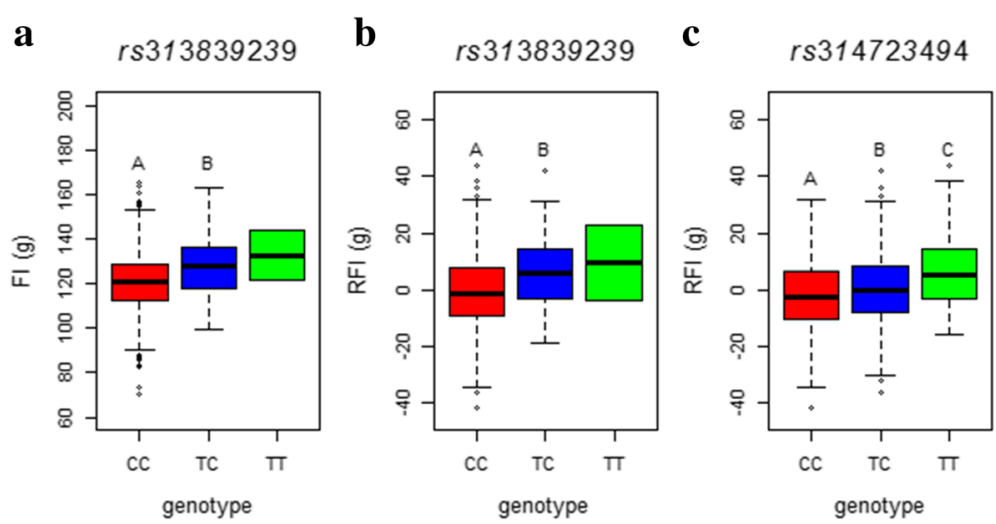

Fig. 3 Boxplot of SNP effect for daily feed intake and residual feed intake. a The effect of rs313839239 on daily feed intake. b The effect of rs313839239 on residual feed intake. c The effect of rs314723494 on daily feed intake. Boxes with different letters are significantly $(P<0.05)$ different from each other. FI and RFI denote daily feed intake and residual feed intake, respectively

\section{Target gene prediction for gga-miR-15a}

To investigate the possible mechanism for gga-miR-15a to influence feed efficiency, further bioinformatics analysis was performed. TargetScan and miRDB software was used for target gene prediction for gga-miR-15a, and a total of 196 and 363 (target score $\geq 80$ ) genes were predicted using these two tools, respectively (Additional file 1: Tables S2 and S3). These target genes were pooled to perform pathway analysis on the DAVID platform after which target genes were significantly $(P<0.05)$ enriched to 9 biological pathways (Table 4) against the database of the Kyoto Encyclopedia of Genes and Genomes (KEGG). After Benjamini p-value correction, only the insulin-signaling pathway (gga-04910) was significantly enriched, with 12 target genes involved. Interaction analysis was then performed on these 12 genes one by one using RNA hybrids. Minor free energy (MFE) was selected as an indicator to identify the reliable bind between microRNA and target mRNA. A total of 9 interactions were found between gga-miR$15 \mathrm{a}$ and three target genes with an MFE less than -20 . These 3 genes were forkhead box O1 (FOXO1),
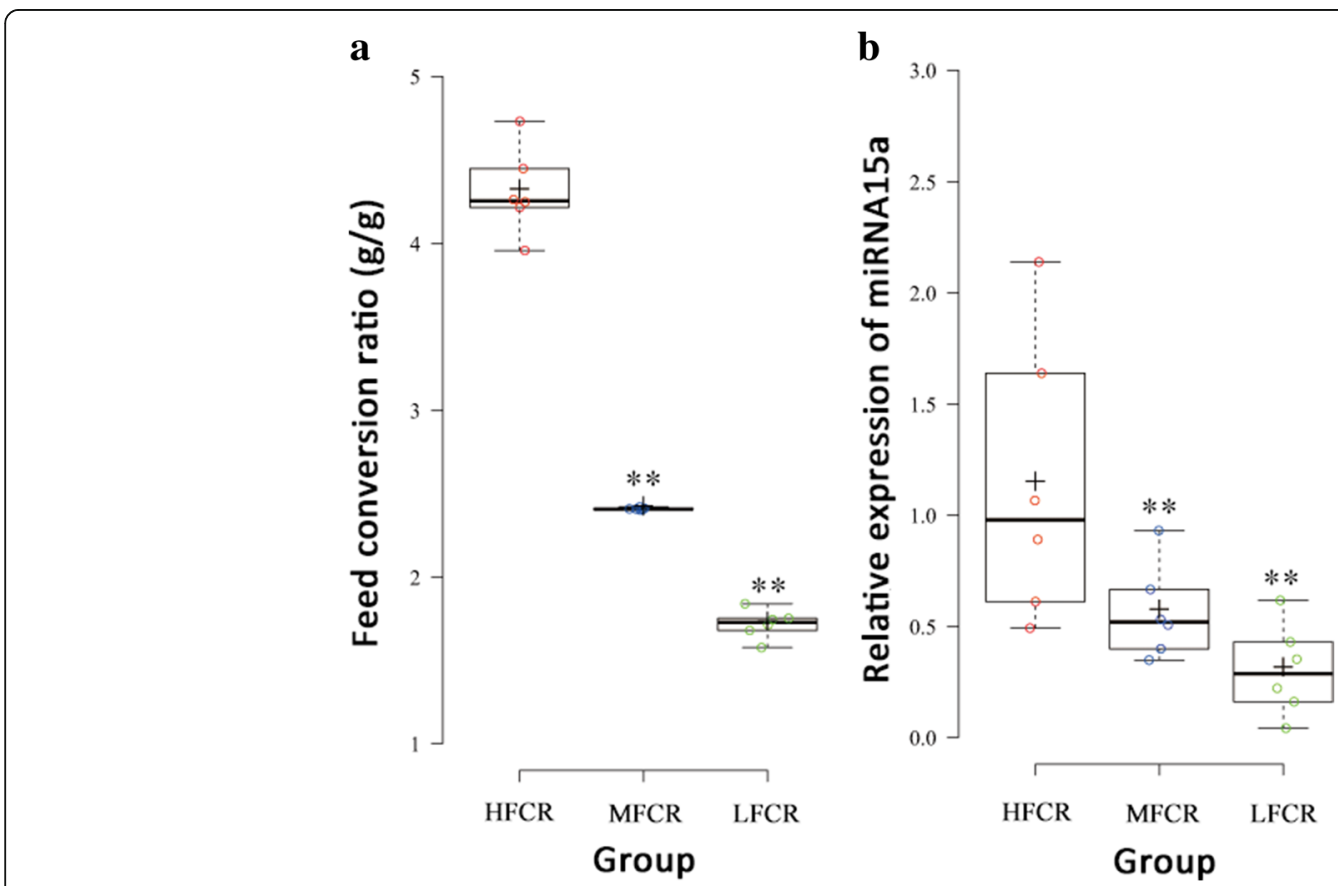

Fig. 4 Expression of gga-mir-15a for hens with high, medium and low feed conversion ratio. a The phenotype of feed conversion ratio for hens selected from high, medium and low feed conversion ratio group. $\mathbf{b}$ Expression of gga-mir-15a for the selected hens. Gene expression is presented relative to $5 \mathrm{~s}$ RNA expression and normalized to a calibrator. ${ }^{*} P<0.01$. Six birds per group were available for the analysis 
Table 4 Significant KEGG pathways for target genes of gga-mir-15a

\begin{tabular}{|c|c|c|c|c|c|}
\hline KEGGa pathway & Count & $\%$ & Involved genes & $P$ value & Benjaminit $^{b}$ \\
\hline Insulin signaling pathway & 12 & 2.48 & $\begin{array}{l}\text { PDPK1, PRKAR2A, CRKL, PHKA1, SOS2, FOXO1, } \\
\text { RAF1, MAPK8, IRS1, INSR, PIK3R1, AKT3 }\end{array}$ & $1.64 \mathrm{E}-03$ & 4.90E-02 \\
\hline mTOR signaling pathway & 8 & 1.65 & $\begin{array}{l}\text { PDPK1, RPS6KA3, ULK1, CAB39, RICTOR, } \\
\text { IRS1, PIK3R1, AKT3 }\end{array}$ & $1.18 \mathrm{E}-03$ & 0.05 \\
\hline FoxO signaling pathway & 13 & 2.69 & $\begin{array}{l}\text { USP7, SGK1, FOXO1, RAF1, IRS1, CCND1, PDPK1, } \\
\text { CDKN2B, SOS2, MAPK8, INSR, AKT3, PIK3R1 }\end{array}$ & $6.79 \mathrm{E}-04$ & 0.06 \\
\hline Oocyte meiosis & 9 & 1.86 & $\begin{array}{l}\text { CCNE1, RPS6KA3, YWHAH, CPEB2, CPEB3, } \\
\text { BTRC, PPP2R5C, YWHAQ, ITPR2 }\end{array}$ & 0.01 & 0.20 \\
\hline MAPK signaling pathway & 15 & 3.10 & $\begin{array}{l}\text { TAOK1, NF1, PPM1A, PTPRR, RAF1, RPS6KA3, } \\
\text { CRKL, MAP3K4, MAP3K2, ELK4, SOS2, MAPK8, } \\
\text { RAPGEF2, NFATC3, AKT33 }\end{array}$ & 0.01 & 0.21 \\
\hline Wnt signaling pathway & 10 & 2.07 & $\begin{array}{l}\text { TBL1XR1, CCND1, NKD1, BTRC, LRP6, MAPK8, } \\
\text { SIAH1, FZD3, NFATC3, WNT7A }\end{array}$ & 0.02 & 0.26 \\
\hline Insulin resistance & 8 & 1.65 & PDPK1, RPS6KA3, FOXO1, MAPK8, IRS1, INSR, PIK3R1, AKT3 & 0.04 & 0.40 \\
\hline Progesterone-mediated oocyte maturation & 7 & 1.45 & RPS6KA3, CPEB2, CPEB3, RAF1, MAPK8, PIK3R1, AKT3 & 0.04 & 0.42 \\
\hline
\end{tabular}

${ }^{a}$ Kyoto Encyclopedia of Genes and Genomes

${ }^{b}$ Benjamini-Hochberg false discovery rate $\leq 0.10$

3-phosphoinositide dependent protein kinase 1 (PDPK1) and protein kinase cAMP-dependent type II regulatory subunit alpha (PRKAR2A) (Additional file 4: Figure S3), and the lowest MFE was found between gga-miR-15a and FOXO1, which showed two binding sites with -28 and $-24.6 \mathrm{kcal} / \mathrm{mol}$ MFE (Fig. 5).

\section{Discussion}

Genetic analysis was conducted in layer chickens affiliated with a nucleus breeding population. Birds were measured for feed intake and efficiency traits at an age greater than 80 weeks. To our knowledge, this study is the first genetic analysis for feed efficiency traits in the late laying period of chickens. Compared with the previous GWAS in the $F_{2}$ resource population [5], the diversity of SNPs was decreased by quality control of minor allele frequency in the current study. This decrease

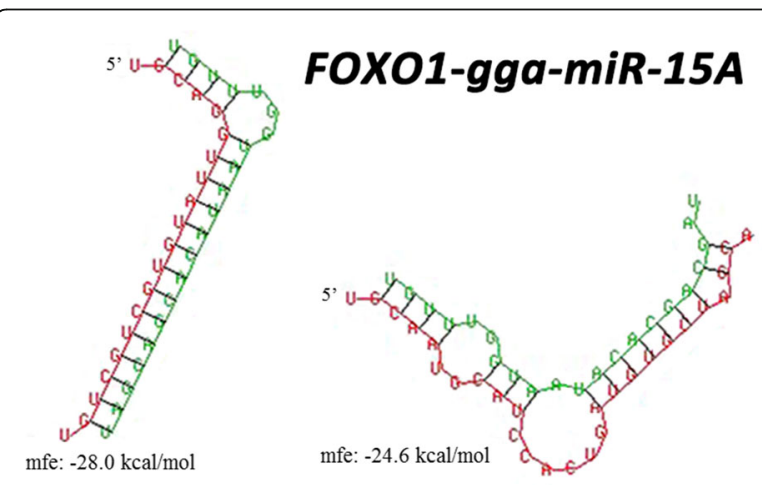

Fig. 5 Molecular interactions between gga-miR-15a and 3 prime untranslated regions (3'-UTR) of FOXO1. Red letters indicate the $3^{\prime}$-UTR sequences of the target genes. Green letters indicate the matured sequences of gga-miR-15a. MFE represents minimal free energy might be due to the long-term selection scheme performed in the nucleus breeding population, which led to many homozygous alleles occurring in the genome. Moreover, the few and different associated hits detected in the current study might be explained by the difference of laying period and population structure [10].

The pedigree-based heritability estimates of FI and RFI presented here were substantially lower than those in the $F_{2}$ population, which were evaluated at the age of 40 and 60 weeks, whereas the estimate of FCR was much higher in the current population. This suggested that different genetic backgrounds could affect the estimates of heritability, as the present study used population that had been selected for egg production for many generations. The high genetic correlation between FI and RFI but not FCR is consistent with our previous findings [11], suggesting that the genetic foundation of RFI is more closely related with that of FI for layer chickens. Additionally, the SNP-based heritability estimates were smaller than pedigree-based estimates, which were likely caused by the "missing heritability" [12] that cannot be explained by common SNPs on the $600 \mathrm{~K} \mathrm{SNP}$ array.

A genomic region of $31 \mathrm{~kb}$ on chromosome 1 (GGA1) that harbored 3 genome-wide significant SNPs was detected associated with the feed conversion ratio (FCR). Additionally, gga-mir-15a (MIR15A) was in this region and close to the three significant SNPs. Given that FCR is related to energy homeostasis and egg production, we constructed cDNA from liver tissue, which is vital to glucose, glucagon, lipid and protein metabolism in chickens [13-15], to conduct a gene expression experiment, i.e., qRT-PCR. The expression of MIR15A was significantly lower in the medium and low FCR birds, which suggested that MIR15A was a promising candidate gene involved in the regulation of FCR. MicroRNAs are small non-coding 
RNAs that have been highly conserved during evolution and have been implicated in multiple molecular interactions [16]. According to the genome of vertebrates, MIR15A, accompanied by MIR16-1 and DELU2 nearby, forms a DLEU2/miR-15a/16-1 cluster to affect chronic lymphocytic leukemia in cancer research $[17,18]$. As an important independent regulatory molecule, microRNA$15 \mathrm{a}$ is involved in the regulation of cell apoptosis and proliferation [19], autoimmunity disease [20, 21], cardiovascular disease [22] and insulin synthesis [23]. Generally, the function of a microRNA is achieved via binding to the 3 prime untranslated region ( $3^{\prime}$-UTR) of target mRNA of a gene [24], with the result that the microRNA represses protein production [25]. Therefore, we used the bioinformatics tools to predict and analyze the target genes of gga-miR-15a, and twelve target genes of gga-miR-15a were significantly enriched in the insulin-signaling pathway. In the chicken liver, the role of the insulin-signaling pathway is similar to that in mammals, which has anabolic effects in glucose transport and utilization, glycogen synthesis, control of liver lipogenic enzymes, amino acid transport and protein synthesis [26]. Sun et al. [23] previously demonstrated that microRNA-15a positively regulated insulin synthesis by targeting uncoupling protein-2 (UCP-2) in mice. In this study, based on the molecular interaction analysis, FOXO1 was identified as the most reliable target gene of gga-mir-15a among 12 target genes. FOXO1, a member of the forkhead box transcription factor class $\mathrm{O}$ (FOXO) family, is a direct transcriptional regulator of gluconeogenesis and glycolysis, reciprocally regulated by insulin, and has profound effects on hepatic lipid metabolism [27]. With the activation of FOXO1, gluconeogenic gene activity is upregulated, promoting glucose production in the liver and accounting largely for the hyperglycemia observed in diabetic individuals [28]. Based on this combined information, we inferred that gga-mir-15a could target FOXO1 by binding to the 3'-UTR of FOXO1 mRNA and then inhibit the protein expression of FOXO1 involved in the insulin-signaling pathway, resulting in the alteration of FCR in chickens.

In the present study, only 5 and 2 hits were identified for FI and RFI at a suggestively significant level, respectively, which indicated that the effect of genetic determinants was too weak to be identified for FI and RFI. Additionally, a pure line selection scheme resulted in effective variants homozygous in the current stocks. However, the suggestively associated SNPs were also promising candidates to some extent. The identified SNPs on chromosome 9 (GGA9) for FI were first reported in chickens according to the QTL database (http://www.animalgenome.org/cgi-bin/QTLdb/GG/index). SNP rs313839239 for RFI was overlapped with a QTL previously identified in commercial meat-type chickens [29]. Notably, because of the high genetic correlation between the two traits, we detected a consensus association (i.e., rs313839239) affecting RFI and FI simultaneously, which is similar to our findings in the laying period from 57 to 60 weeks in a previous study [5]. FARP2 (FERM, RhoGEF and pleckstrin domaincontaining protein 2), a guanine nucleotide exchange factor in the Rho family of small GTPases, was a shared candidate gene for both FI and RFI. FARP2 was identified as a candidate gene in diabetes research [30], and is correlated with energy metabolism and obesityassociated pathologies [31]. Another promising candidate gene for RFI was cannabinoid receptor 1 (CNR1), which is referred to in an energy homeostasis and metabolic process revealed by pharmacological studies [32].

\section{Conclusions}

In conclusion, the screened genomic region/variants for FCR, FI and RFI can be valuable references for designing the customized genetic and genomic selection schemes to improve efficiency of feed utilization in the current nucleus of breeding flocks. MIR15A should be considered a primary candidate gene to improve the understanding of the genetic and physiological factors affecting the FCR in investigated populations. The interactions between MIR15A and target genes, such as FOXO1, suggested that the insulin-signaling pathway in the liver might be the causative factor affecting FCR regulation in chickens. The mechanisms by which these relevant factors modulate metabolism and homeostasis in the pathway should be studied further in chickens. This new insight provided by our study will help to understand the biological regulation of metabolism and homeostasis in chickens.

\section{Materials and methods \\ Animals and phenotypes}

Female chickens from the 11th generation of a pedigreed line of Rhode Island Red were maintained by Beijing Huadu Yukou Poultry Breeding Co. Ltd., China with selection on egg production and quality. Birds were generated from the same hatch and housed in identically individual cages with free access to feed and water. Feed intake was individually collected during a 2-wk. (8182 weeks of age) trial period. In the 2 -wk. feeding test period, feed was provided in individual containers for each hen. Feed consumption data were procured daily by manual collection to derive the daily feed intake (FI). Egg mass (EM) was obtained as total egg number multiplied by average egg weight for each hen. Daily egg number was recorded to calculate total egg number, and the weight of 3 consecutive eggs collected in each week was used to calculate average egg weight. Body weight was measured on a mid-test day for each recorded hen. 
Feed conversion ratio (FCR) was calculated as the ratio of FI and daily EM. After removing outliers (values greater than $3 \mathrm{SD}$ from the mean) for FCR, the remaining 808 hens were used to calculate residual feed intake (RFI) as the residuals from a regression model of FI on EM and metabolic BW (BW raised to the power of 0.75) [33]. The phenotypes that did not follow a normal distribution were transformed by Johnson method implemented in $\mathrm{R}$ software, and then transformed data were used in the next genetics analysis.

\section{Genotyping, imputation and quality control}

Genomic DNA was isolated from whole blood samples using phenol-chloroform methods. The qualified 808 hens were genotyped for 580,961 markers using Affymetrix $600 \mathrm{~K}$ chicken high-density genotyping array. In the quality control of raw data, all individuals passed the criteria with a missing SNP call rate $<5 \%$ using Affymetrix power tool (APT) provided by Affymetrix (http:// www.affymetrix.com/). Autosomal SNPs of 808 samples were filtered by the criteria set in PLINK [34] (sample call rate $>97 \%$, minor allele frequencies $>1 \%$ and Hardy Weinberg equilibrium $P$-value $<1 \mathrm{e}-6)$. Afterward, the remaining SNPs and 808 birds were used for the imputation implemented in the Beagle Version 4 software package based on localized haplotype clustering [35]. Finally, a total of 307,216 SNPs distributed on 28 autosomes and 2 linkage groups, listed in Tables 5, and 808 birds were obtained for subsequent genetic analyses after filtering for imputation results using PLINK.

\section{Evaluation of genetic parameters}

Prior to the genetic analysis, the effect cage tiers were tested using analysis of variance implemented in SAS software, and effect of cage tiers was excluded from the next analysis due to lack of the significance. Pedigreebased genetic parameters for FI, RFI and FCR were estimated with the average information restricted maximum likelihood (AI-REML) method implemented in DMU software [36]. The multi-trait animal model adopted in the current analysis was the following:

$$
\mathrm{y}=1 \mu+\mathrm{Za}+\mathrm{e}
$$

where $\mathrm{y}$ is the phenotypic value of each trait; 1 and $\mathrm{Z}$ are the incidence matrix of fixed effects (population means) and random effects (individual additive genetic effect), respectively; $\mu$ is the vector of fixed effects of population means; and a and e are the random additive effects and residual effect, respectively. Estimation of the phenotypic variance explained by significantly associated SNPs and all SNPs (SNP-based heritability [37] and SNP-based genetic correlation [38]) was calculated by restricted maximum likelihood (REML) analysis implemented in GCTA software [39].

\section{Genome-wide association analysis}

Before the genome-wide association study (GWAS), the eligible SNPs and birds were used to evaluate the population structure by PLINK. First, all SNPs were pruned to obtain independent SNP markers using the indep-

Table 5 Basic information for SNP markers on a physical map after quality control

\begin{tabular}{llllllll}
\hline Chromosome & Map distance $(\mathrm{Kb})^{\mathrm{a}}$ & No. SNPs & Density (kb/SNP) & Chromosome & Map distance (Kb) & No. SNPs & Density (kb/SNP) \\
\hline 1 & $195,241.9$ & 58,459 & 3.3 & 16 & 494.8 & 266 & 1.9 \\
2 & $148,556.6$ & 35,247 & 4.2 & 17 & $10,279.4$ & 4954 & 2.1 \\
3 & $110,445.2$ & 33,201 & 3.3 & 18 & $11,198.7$ & 5560 & 2.0 \\
4 & $90,168.3$ & 26,060 & 3.5 & 19 & 9979.3 & 4911 & 2.0 \\
5 & $59,540.0$ & 17,745 & 3.4 & 20 & $14,252.9$ & 4843 & 2.9 \\
6 & $34,904.9$ & 11,610 & 3.0 & 21 & 6786.7 & 4808 & 1.4 \\
7 & $36,195.7$ & 12,537 & 2.9 & 22 & 4050.3 & 2234 & 1.8 \\
8 & $28,724.0$ & 9276 & 3.1 & 23 & 5700.7 & 3508 & 1.6 \\
9 & $23,424.5$ & 11,201 & 2.1 & 24 & 6313.8 & 4505 & 1.4 \\
10 & $19,856.0$ & 9704 & 2.0 & 25 & 2188.5 & 1494 & 1.5 \\
11 & $19,381.0$ & 7563 & 2.6 & 26 & 5288.3 & 3465 & 1.5 \\
12 & $19,844.9$ & 7772 & 2.6 & 27 & 5143.2 & 2922 & 1.8 \\
13 & $17,425.5$ & 6088 & 2.9 & 28 & 4735.4 & 2845 & 1.7 \\
14 & $15,145.4$ & 8102 & 1.9 & LGE64 & 953.8 & 94 & 10.1 \\
15 & $12,624.9$ & 6203 & 2.0 & LGE22 & 739.1 & 39
\end{tabular}

${ }^{a}$ The physical length of the chromosome was based on the position of the last marker in the Gullus gullus version 4

bLGE22, linkage group LGE22C19W28_E50C23 
pairwise option, with a window size of 25 SNPs, a step of 5 SNPs, and $r^{2}$ threshold of 0.2. Second, pairwise identity-by-state (IBS) distances were calculated between all individuals using the independent SNP markers. Finally, we calculated multidimensional scaling (MDS) components using the mds-plot option based on the IBS matrix, which was included as a covariate in the subsequent association analyses. GWAS was performed using a mixed models approach [40] implemented in the GEMMA software package, which fitted a linear mixed model to account for population stratification and sample structure with a faster computational time [41]. Association test with univariate linear mixed model (univariate GWAS) was performed for each trait. The statistical model was the following:

$$
\mathrm{y}=\mathrm{W} \alpha+\mathrm{x} \beta+\mathrm{u}+\varepsilon
$$

where $\mathrm{y}$ is the vector of traits value for all individuals; W is a matrix of covariates (fixed effects contain first 4 MDS components and a column of $1 \mathrm{~s}$ ); $\alpha$ is a vector of the corresponding coefficients including the intercept; $x$ is a vector of marker genotypes; $\beta$ is the effect size of the marker; $\mathrm{u}$ is a vector of individual random effects; $\varepsilon$ is vector of errors. The Wald test statistic $P$-value was used as the criterion to screen SNPs associated with the investigated traits. Conditional GWA analyses were performed using the same mixed model with the addition of the dosage of the strongest associated SNP as a covariate [42].

\section{Statistical and bioinformatics analyses}

With respect to the $P$-value threshold of genome-wide significance, the simpleM method [43] was used to infer the independent tests. A total of 38,715 independent tests over the entire autosomal SNPs were obtained, and then genome-wide significance and suggestive significance were calculated as $1.29 \mathrm{e}-6(0.05 / 38,715)$ and $2.58 \mathrm{e}-5(1.00 / 38,715)$, respectively. The Manhattan and Q-Q plots were constructed for each trait by the GAP package (http://cran.r-project.org/web/packages/gap/ index.html) within the $\mathrm{R}$ software. Linkage disequilibrium (LD) analysis was performed for the chromosomal regions with many associated SNPs clustered implemented in Haploview version 4.2 [44] with the algorithm proposed by Gabriel et al. [45].

SNP positions and information were obtained using annotation of Gallus gallus 4.0 genome version, and genes within 500,000 base pairs flanking the associated SNPs were chosen for further analysis. Target genes of microRNA were predicted using TargetScan (http:// www.targetscan.org) and miRDB (http://www.mirdb.org/ miRDB/). Kyoto Encyclopedia of Genes and Genomes (KEGG) pathway analysis was used to analyze target genes of microRNA implemented online with the DAVID platform (https://david.ncifcrf.gov/). Bioinformatics analyses of molecular interactions between microRNA and predicted coding genes were performed using RNAhybrid online [46] in which minimal free energy (MFE) of interaction less than -20 was considered binding. The mature miRNA sequence was downloaded from miRBase (http://www.mirbase.org/), and the target sequence of coding genes was queried from Ensembl online (http://www.ensembl.org/).

\section{Total RNA extraction and qRT-PCR}

Birds were deeply anesthetized with sodium pentobarbital via cardiopuncture, and decapitated. Liver tissue samples were collected from chickens along with the regular quarantine inspection of the experimental station of China Agricultural University in accordance with the Guidelines for the Care and Use of Experimental Animals established by the Ministry of Agriculture of China (Beijing, China). The entire study was approved by the Animal Welfare Committee of China Agricultural University (permit number: SYXK 2007-0023).

Total RNA was extracted from the liver tissue using a mirVana $^{\mathrm{Tm}}$ miRNA Isolation Kit (Life Technologies, Carlsbad, CA, USA), and was reversely transcribed using a miRACLE cDNA Synthesis Kit (Genetimes Technology, Shanghai, China) as described by the manufacturer. The quantitative real-time PCR (qRT-PCR) was performed on an ABI 7500 system (Applied Biosystems). Primers of ggamiR-15a and chicken $5 \mathrm{~s}$ rRNA were designed and synthesized by Genetimes Technology Inc. (Shanghai, China). The mature miRNAs were polyadenylated by polyA polymerase and reversely transcribed into complementary DNA (cDNA) using a reverse primer, which had a 3 prime degenerate anchor and a universal tag sequence on the 5 prime end. The cDNA template was then amplified using specific forward and universal reverse primers. The specific forward primer sequences of the miRNAs used in this study were the following: gga-miR-15a, forward 5'TAGCAGCACATAATGGTTTGTAAAA-3' and chicken 5 s rRNA, forward 5'-ACCGGGTGCTGTAGGCTTAA$3^{\prime}$. The universal reverse primer was included in the qPCR Kit of miRACLE qPCR miRNA Master Mix (Genetimes Technology). The optimum thermal cycling conditions were as follows: $95^{\circ} \mathrm{C}$ for $10 \mathrm{~min}, 40$ cycles of $95{ }^{\circ} \mathrm{C}$ for $10 \mathrm{~s}, 60{ }^{\circ} \mathrm{C}$ for $20 \mathrm{~s}, 72^{\circ} \mathrm{C}$ for $1 \mathrm{~min}, 95^{\circ} \mathrm{C}$ for $15 \mathrm{~s}, 60{ }^{\circ} \mathrm{C}$ for $30 \mathrm{~s}$, and $95{ }^{\circ} \mathrm{C}$ for $15 \mathrm{~s}$. All experiments were run in triplicate. Relative quantification of microRNA expression was analyzed using the $\Delta \Delta C T$ method [47] with 5 s RNA as the endogenous control and the average of the birds in HFCR group as the calibrator sample. Relative quantities calculated as $2^{-\Delta \Delta C T}$ were used for statistical analyses. Data were analyzed by pairwise Student's t-tests implemented in the $\mathrm{R}$ software. 


\section{Additional files}

Additional file 1: Table S1. The information for SNPs suggestively associated with feed conversion ratio. Table S2. Predicted target genes from TargetScan for gga-mir-15a. Table S3. Predicted target genes from miRDB for gga-mir-15a. (XLSX 37 kb)

Additional file 2: Figure S1. Linkage map of the associated genomic region for daily feed intake on chicken chromosome 9. (TIFF 166 kb)

Additional file 3: Figure S2. Regional association plot for FCR after conditional analysis on rs 13553102. The graph plots genomic position ( $x$ axis) against its significance expressed as $-\log 10 P$ value (y axis). (TIFF 205 kb)

Additional file 4: Figure S3. Molecular interactions between gga-mir$15 \mathrm{a}$ and target genes with minimal free energy less than $-20 \mathrm{kcal} / \mathrm{mol}$. Red letters indicate the 3' UTR sequences of the target genes. Green letters indicate the matured sequences of gga-miR-15a. (TIFF $3113 \mathrm{~kb}$ )

\section{Abbreviations}

AI-REML: Average information restricted maximum likelihood: DAVID: Database for annotation, visualization and integrated discovery; EM: Daily egg mass; FCR: Feed conversion ratio; Fl: Daily feed intake; GGA: Gallus gallus chromosome; GIF: Genomic inflation factors; GWAS: Genome-wide association study; KEGG: Kyoto Encyclopedia of Genes and Genomes; LD: Linkage disequilibrium; MAF: Minor allele frequency; MBW: Mean body weight; MFE: Minor free energy; qRT-PCR: Quantitative real time polymerase chain reaction; RFI: Residual feed intake; SNP: Single nucleotide polymorphism

\section{Acknowledgements}

We thank Beijing HuaduYukou Poultry Industry Co. Ltd. for providing chickens used in the molecular experiment.

\section{Funding}

This work was funded in part by the National Natural Science Foundation of China (31501915) in experiment design and data acquisition; by Programs for Changjiang Scholars and Innovative Research in Universities (IRT_15R62) for data analysis, and by China Agriculture Research Systems (CARS-40) in manuscript writing and edit.

\section{Availability of data and materials}

The datasets used and/or analysed during the current study are available from the corresponding author on reasonable request.

\section{Authors' contributions}

CS conceived the study and designed the project. JY performed genetic, bioinformatics and molecular analyses. JY, SC, FS, GW, and AL contributed to collecting samples and measuring phenotypic data. JY wrote the manuscript, NY and CS revised the manuscript. All authors read and approved the final manuscript.

\section{Ethics approval and consent to participate}

Blood and liver tissue samples were collected from chickens along with the regular quarantine inspection of the experimental station of China Agricultural University in accordance with the Guidelines for the Care and Use of Experimental Animals established by the Ministry of Agriculture of China (Beijing, China). The entire study was approved by the Animal Welfare Committee of China Agricultural University (permit number: SYXK 2007-0023).

\section{Consent for publication}

Not applicable

\section{Competing interests}

The authors declare that they have no competing interests.

\section{Author details}

${ }^{1}$ National Engineering Laboratory for Animal Breeding and MOA Key Laboratory of Animal Genetics and Breeding, College of Animal Science and Technology, China Agricultural University, Beijing, China. ${ }^{2}$ Beijing Engineering Research Center of Layer, Beijing, China.
Received: 27 March 2017 Accepted: 28 August 2017

Published online: 06 September 2017

\section{References}

1. Patience JF, Rossoni-Serão MC, Gutiérrez NA. A review of feed efficiency in swine: biology and application. J Anim Sci Biotechno. 2015;6(1):33.

2. Wang Z, Qu L, Yao J, Yang X, Li G, Zhang Y, Li J, Wang X, Bai J, Xu G, et al. An EAV-HP insertion in $5^{\prime}$ flanking region of SLCO1B3 causes blue eggshell in the chicken. PLoS Genet. 2013;9(1):e1003183.

3. Ma J, Yang J, Zhou L, Ren J, Liu X, Zhang H, Yang B, Zhang Z, Ma H, Xie X, et al. A splice mutation in the PHKG1 gene causes high glycogen content and low meat quality in pig skeletal muscle. PLOS Genet. 2014;10(10):e1004710.

4. Bolormaa S, Pryce JE, Reverter A, Zhang Y, Barendse W, Kemper K, Tier B, Savin K, Hayes BJ, Goddard ME. A multi-trait, meta-analysis for detecting pleiotropic polymorphisms for stature, fatness and reproduction in beef cattle. PLoS Genet. 2014;10(3):e1004198.

5. Yuan J, Wang K, Yi G, Ma M, Dou T, Sun C, Qu L-J, Shen M, Qu L, Yang N. Genome-wide association studies for feed intake and efficiency in two laying periods of chickens. Genes Sel Evol. 2015;47(1):82.

6. Mucha S, Grajewski B, Gornowicz E, Lisowski M, Radziszewska J, Szwaczkowski T. Mapping quantitative trait loci affecting some carcass and meat traits in duck (Anas Platyrhynchos). J Appl Genet. 2014:55(4):497-503.

7. Minvielle F, Kayang BB, Inoue-Murayama M, Miwa M, Vignal A, Gourichon D, Neau A, Monvoisin J-L. Ito Si: microsatellite mapping of QTL affecting growth, feed consumption, egg production, tonic immobility and body temperature of Japanese quail. BMC Genomics. 2005;6(1):87.

8. Minvielle F, Kayang BB, Inoue-Murayama M, Miwa M, Vignal A, Gourichon D, Neau A, Monvoisin J-L, Shin'ichi I. Microsatellite mapping of QTL affecting growth, feed consumption, egg production, tonic immobility and body temperature of Japanese quail. BMC Genomics. 2005;6(1):87.

9. Bain MM, Nys Y, Dunn IC. Increasing persistency in lay and stabilising egg quality in longer laying cycles. What are the challenges? Brit Poultry Sci. 2016;57(3):330-8

10. Fu W, Dekkers JCM, Lee WR, Abasht B. Linkage disequilibrium in crossbred and pure line chickens. Genet Sel Evol. 2015;47(1):11.

11. Yuan J, Dou T, Ma M, Yi G, Chen S, Qu L, Shen M, Qu L, Wang K, Yang N. Genetic parameters of feed efficiency traits in laying period of chickens. Poultry Sci. 2015;94(7):1470-5.

12. Manolio TA, Collins FS, Cox NJ, Goldstein DB, Hindorff LA, Hunter DJ, McCarthy MI, Ramos EM, Cardon LR, Chakravarti A, et al. Finding the missing heritability of complex diseases. Nature. 2009;461(7265):747-53.

13. Buyse J, Decuypere E. Chapter 19 - adipose tissue and lipid metabolism A2 Scanes, Colin G. In: Sturkie's Avian physiology. 6th ed. San Diego: Academic Press; 2015. p. 443-53.

14. Scanes CG. Chapter 18 - carbohydrate metabolism. In: Sturkie's Avian physiology. 6th ed. San Diego: Academic Press; 2015. p. 421-41.

15. Scanes CG. Chapter 20 - protein metabolism. In: Sturkie's Avian physiology. 6th ed. San Diego: Academic Press; 2015. p. 455-67.

16. Carrington JC, Ambros V. Role of MicroRNAs in plant and animal development. Science. 2003;301(5631):336

17. Calin GA, Cimmino A, Fabbri M, Ferracin M, Wojcik SE, Shimizu M, Taccioli C, Zanesi N, Garzon R, Ageilan Rl, et al. MiR-15a and miR-16-1 cluster functions in human leukemia. P Natl Acad Sci USA. 2008;105(13):5166-71.

18. Klein U, Lia M, Crespo M, Siegel R, Shen Q, Mo T, Ambesi-Impiombato A, Califano A, Migliazza A, Bhagat G, et al. The DLEU2/miR-15a/16-1 cluster controls $B$ cell proliferation and its deletion leads to chronic lymphocytic leukemia. Cancer Cell. 2010;17(1):28-40.

19. Andersen DC, Jensen $\mathrm{CH}$, Schneider M, Nossent AY, Eskildsen T, Hansen JL, Teisner B, Sheikh SP. MicroRNA-15a fine-tunes the level of Delta-like 1 homolog (DLK1) in proliferating 3T3-L1 preadipocytes. Exp Cell Res. 2010;316(10):1681-91.

20. Yuan Y, Kasar S, Underbayev C, Vollenweider D, Salerno E, Kotenko SV, Raveche E. Role of microRNA-15a in autoantibody production in interferon-augmented murine model of lupus. Mol Immunol. 2012:52(2):61-70.

21. Chatzikyriakidou A, Voulgari PV, Georgiou I. Drosos AA: miRNAs and related polymorphisms in rheumatoid arthritis susceptibility. Autoimmun Rev. 2012;11(9):636-41. 
22. Spinetti G, Fortunato O, Caporali A, Shantikumar S, Marchetti M, Meloni M, Descamps B, Floris I, Sangalli E, Vono R, et al. MicroRNA-15a and MicroRNA16 impair human circulating proangiogenic cell functions and are increased in the proangiogenic cells and serum of patients with critical limb ischemia. Circ Res. 2013;112(2):335.

23. Sun L-L, Jiang B-G, Li W-T, Zou J-J, Shi Y-Q, Liu Z-M. MicroRNA-15a positively regulates insulin synthesis by inhibiting uncoupling protein-2 expression. Diabetes Res Clin Pract. 2011;91(1):94-100.

24. Bartel DP. MicroRNAs: target recognition and regulatory functions. Cell. 2009;136(2):215-33.

25. Cannell lan G, Kong Yi W, Bushell M. How do microRNAs regulate gene expression? Biochem Soc Trans. 2008;36(6):1224.

26. Dupont J, Tesseraud S, Simon J. Insulin signaling in chicken liver and muscle. Gen Comp Endocrinol. 2009;163(1-2):52-7.

27. Tikhanovich I, Cox J, Weinman SA. Forkhead box class $O$ transcription factors in liver function and disease. J Gastroenterol Hepatol. 2013;28:125-31.

28. Cheng Z, White MF. The AKTion in non-canonical insulin signaling. Nat Med. 2012;18(3):351-3.

29. De Koning DJ, Haley CS, Windsor D, Hocking PM, Griffin H, Morris A, Vincent J, Burt DW. Segregation of QTL for production traits in commercial meattype chickens. Genet Res. 2004;83(03):211-20.

30. Pezzolesi MG, Poznik GD, Mychaleckyj JC, Paterson AD, Barati MT, Klein JB, Ng DPK, Placha G, Canani LH, Bochenski J et al.: Genome-wide association scan for diabetic nephropathy susceptibility genes in type 1 diabetes. Diabetes 2009, 58(6):1403.

31. Chang Y-J, Pownall S, Jensen TE, Mouaaz S, Foltz W, Zhou L, Liadis N, Woo M, Hao Z, Dutt $P$, et al. The rho-guanine nucleotide exchange factor PDZRhoGEF governs susceptibility to diet-induced obesity and type 2 diabetes. elife. 2015;4:e06011.

32. Cristino L, Becker T, Di Marzo V. Endocannabinoids and energy homeostasis: an update. Biofactors. 2014;40(4):389-97.

33. Wolc A, Arango J, Jankowski T, Settar P, Fulton JE, O'Sullivan NP, Fernando R, Garrick DJ, Dekkers JCM. Pedigree and genomic analyses of feed consumption and residual feed intake in laying hens. Poultry Sci. 2013;92(9):2270-5.

34. Purcell S, Neale B, Todd-Brown K, Thomas L, Ferreira MAR, Bender D, Maller J, Sklar P, de Bakker PIW, Daly MJ, et al. PLINK: a tool set for whole-genome association and population-based linkage analyses. J Hum Genet. 2007;81(3):559-75.

35. Browning SR, Browning BL. Rapid and accurate haplotype phasing and missing-data inference for whole-genome association studies by use of localized haplotype clustering. The J Hum Genet. 2007;81(5):1084-97.

36. Madsen $P$, Jensen J: DMU: A user's guide. A package for analysing multivariate mixed models. Version 6, release 4.7. In 2007.

37. Yang J, Benyamin B, McEvoy BP, Gordon S, Henders AK, Nyholt DR, Madden PA, Heath AC, Martin NG, Montgomery GW, et al. Common SNPs explain a large proportion of the heritability for human height. Nat Genet. 2010;42(7):565-9.

38. Lee SH, Yang J, Goddard ME, Visscher PM, Wray NR. Estimation of pleiotropy between complex diseases using SNP-derived genomic relationships and restricted maximum likelihood. Bioinformatics. 2012;28(19):2540-2.

39. Yang J, Lee SH, Goddard ME, Visscher PM. GCTA: a tool for genome-wide complex trait analysis. Am J Hum Genet. 2011;88(1):76-82.

40. Price AL, Zaitlen NA, Reich D, Patterson N. New approaches to population stratification in genome-wide association studies. Nat Rev Genet. 2010;11(7):459-63.

41. Zhou X, Stephens M. Genome-wide efficient mixed-model analysis for association studies. Nat Genet. 2012;44(7):821-4.

42. Schizophrenia Psychiatric Genome-Wide Association Study C. Genome-wide association study identifies five new schizophrenia loci. Nat Genet. 2011;43(10):969-76.

43. Gao X. Multiple testing corrections for imputed SNPs. Genet Epidemiol. 2011;35(3):154-8.

44. Barrett JC, Fry B, Maller J, Daly MJ. Haploview: analysis and visualization of LD and haplotype maps. Bioinformatics. 2005;21(2):263-5.
45. Gabriel SB, Schaffner SF, Nguyen H, Moore JM, Roy J, Blumenstiel B, Higgins J, DeFelice M, Lochner A, Faggart M, et al. The structure of haplotype blocks in the human genome. Science. 2002;296(5576):2225.

46. Rehmsmeier M, Steffen $P$, Höchsmann M, Giegerich R. Fast and effective prediction of microRNA/target duplexes. RNA. 2004;10(10):1507-17.

47. Livak KJ, Schmittgen TD. Analysis of relative gene expression data using real-time quantitative $P C R$ and the $2-\Delta \Delta C T$ method. Methods. $2001 ; 25(4): 402-8$

\section{Submit your next manuscript to BioMed Central and we will help you at every step:}

- We accept pre-submission inquiries

- Our selector tool helps you to find the most relevant journal

- We provide round the clock customer support

- Convenient online submission

- Thorough peer review

- Inclusion in PubMed and all major indexing services

- Maximum visibility for your research

Submit your manuscript at www.biomedcentral.com/submit 